\title{
Spontaneous hemothorax and neurofibromatosis type 1: How to explain it, how to explore it and how to treat it?
}

\author{
Monia Attia ${ }^{1,2}$, Houda Gharsalli ${ }^{2,3}$, Haifa Rmilii ${ }^{1,2}$, Meriem Affes ${ }^{1,2}$, Soumaya Ben Saad ${ }^{2,3}$, Sarra Zairi ${ }^{2,4}$, \\ Ines Baccouche ${ }^{1,2}$, Saoussen Hantous ${ }^{1,2}$, HendaNeji ${ }^{1,2}$, Khaoula Ben Miled-M'rad ${ }^{1,2}$ \\ ${ }^{1}$ Department of Radiology, Abderrahmane Mami Hospital, Ariana; ${ }^{2}$ Faculty of Medicine, University El Manar of \\ Tunis; ${ }^{3}$ Department of Pulmonology, Abderrahmane Mami Hospital, Ariana; ${ }^{4}$ Department of Thoracic and \\ Cardiovascular Surgery, Abderrahmane Mami Hospital, Ariana, Tunisia
}

\begin{abstract}
Neurofibromatosis type 1 (NF1), also known as Von Recklinghausen's disease is an autosomal dominant genetic disorder. It is the most common of phacomatoses. Pulmonary complications have been rarely described in the literature. It is generally a benign disease, but has the potential for rare and fatal complications, such as spontaneous hemothorax. We reported two cases of patients with a history of von Recklinghausen's disease. Both of them suffered sudden chest pain. Chest-X ray revealed a pan hemi-thoracic opacity. Enhanced chest computed tomography showed massive hemothorax, but no evidence of tumors or an obvious bleeding point in the thorax. After we had ensured a stable hemodynamic condition, we performed video-assisted thoracic surgery to remove the hematoma. No evidence of bleeding was noticed in the first patient whereas an active bleeding was observed in the second patient. We could not determine which vessel was responsible of the hemorrhage. Electrocoagulation and clot removal were performed. Evolution was favorable for both patients. Spontaneous hemothorax is a rare and potentially life threatening NF1's complication. A tumor like neurofibroma or more rarely vascular involvement of large or small caliber arteries may be at hemothorax's origin.
\end{abstract}

Corresponding author: Monia Attia, Department of Radiology, Abderrahmane Mami Hospital, Rue de l'Hôpital, 2080 Ariana, Tunisia. E-mail: attiamonia82@gmail.com

Key words: Spontaneous hemothorax; neurofibromatosis.

Conflict of interest: The authors declare no conflict of interest.

Funding: There is no funding associated with this manuscript.

Received for publication: 20 January 2019.

Accepted for publication: 15 May 2019.

(C) Copyright M. Attia et al., 2019

Licensee PAGEPress, Italy

Monaldi Archives for Chest Disease 2019; 89:1038

doi: 10.4081/monaldi.2019.1038

This article is distributed under the terms of the Creative Commons Attribution Noncommercial License (by-nc 4.0) which permits any noncommercial use, distribution, and reproduction in any medium, provided the original author(s) and source are credited.

\section{Introduction}

Neurofibromatosis type 1 (NF1), also known as Von Recklinghausen's disease, is the most common of phacomatoses. It is transmitted according to an autosomic dominant mode and presents a quasi-complete penetrance at the age of 8 years. The gene responsible for the disease, a suppressive tumor gene, is located on the long arm of chromosome 17 in 17q11.2 [1,2].

NF1 is characterized by polymorphic clinical presentations, most frequent are cutaneous and neurological manifestations [1]. Vascular lesions are less well known during NF1 reported in only 1 to $3 \%$ of patients [3]. They may interest large and medium-sized vessels as aneurysm or stenosis. Indeed, renal artery stenosis represents the most frequent manifestation [3]. Involvement of small caliber vessels is less known and may lead to spontaneous hemorrhagic complications.

We report observations of two patients with NF1 admitted for the management of a great abundance hemothorax.

\section{Case Reports}

The first patient was a 57-year-old man, followed for a familial NF1, who presented to the emergency department for left chest pain that had appeared for $48 \mathrm{~h}$ and intensified abruptly.

In addition to café-au-lait spots and diffuse skin neurofibromas, clinical examination noted polypnea at $20 \mathrm{c} / \mathrm{min}$, heart rate at $100 \mathrm{bpm}$, blood pressure at $110 / 80 \mathrm{mmHg}, 90 \% \mathrm{O}_{2}$ saturation as well as a left pleural effusion syndrome. The biological assessment showed a hemoglobin level of $13.5 \mathrm{~g} / \mathrm{dl}$ and a prothrombin (PT) level of $62 \%$.

Chest X-ray showed an opacity occupying the entire left lung field (Figure 1). The diagnosis of a compressive great abundance pleural effusion was carried. Thoracocentesis was practiced bringing back a frankly hematic liquid. In order to seek the cause of bleeding, an angio-CT scan was immediately indicated. This examination was performed before and after contrast injection at pulmonary and aortic times. It demonstrated a left pleural effusion with a spontaneously hyperdense component (Figure 2). After injection of iodinated contrast product, no extravasation was detected. Computed tomography (CT) showed also thoracic scoliosis, central spinal canal and left foramen enlargement, extending from T1 to T4. The patient was referred to thoracic surgery department where he underwent a thoracoscopy with clot removal and chest drainage. Surgeon did not find any arterial bleeding or tumor which could 
have caused this hemothorax. Evolution was favorable clinically and radiologically with removal of the drain at the fourth postoperative day. Magnetic resonance imaging (MRI) was considered necessary to explore the enlargement of thoracic spinal canal and lateral foramens detected by CT. It showed thoracic dural ectasia without any neurofibroma or other mesenchymal tumor (Figure 3).

The second case concerned a 31-year-old female patient with a history of kyphoscoliosis surgery who had acute right chest pain with rapid worsening dyspnea, becoming stage IV. No clinical signs or trauma prior to this episode have been reported. Physical examination revealed multiple café-au-lait spots as well as soft skin growths. The patient was hemodynamically stable. However, she had a polypnea at 40 cycles/min and a right pleural effusion syndrome. Peripheral oxygen saturation was $89 \%$. Chest X-ray revealed a white right hemithorax (Figure 4). Initial hemoglobin was $12.5 \mathrm{~g} / \mathrm{dl}$. Based on clinical and radiological data, pleural effusion has been suspected. It was confirmed by a pleural thoracocen-

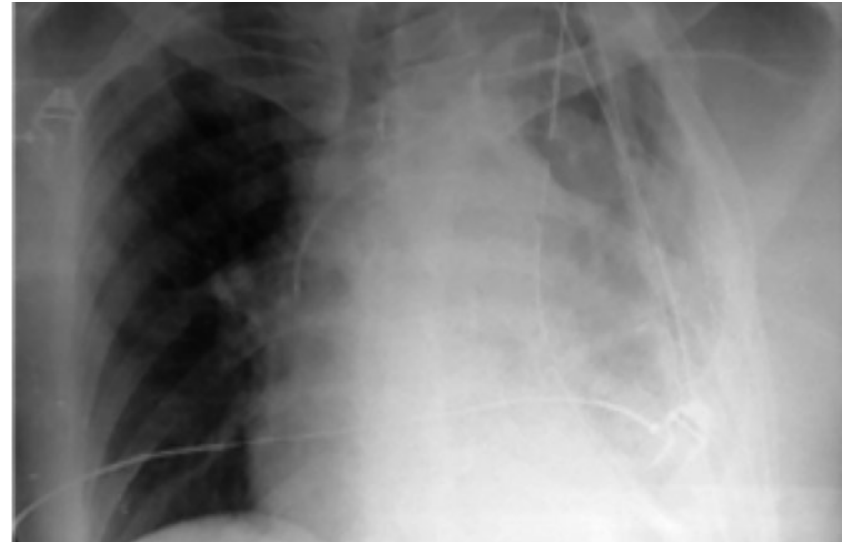

Figure 1. Chest X-ray: opacity involving left hemithorax.
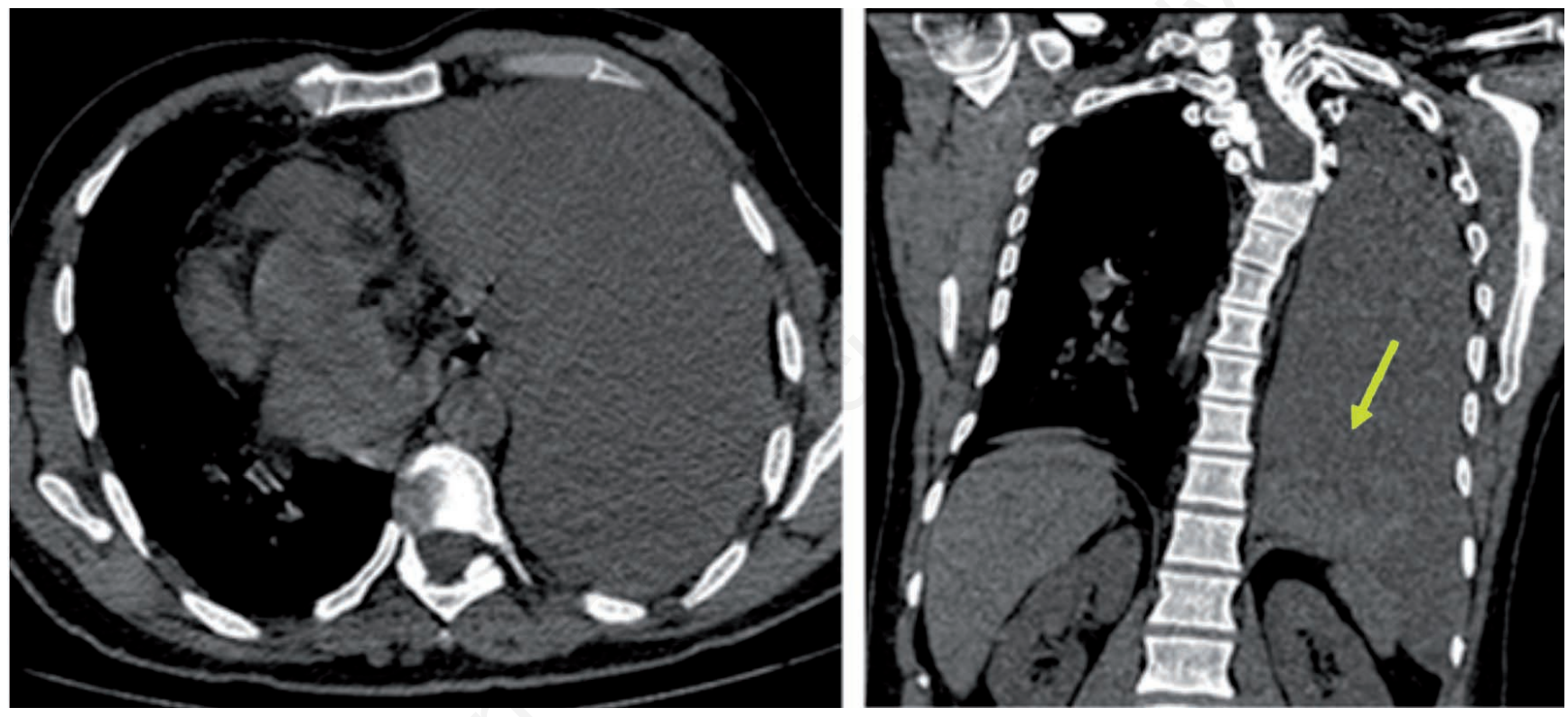

Figure 2. Chest CT scan without contrast injection: left pleural effusion with a spontaneously hyperdense component (hemothorax) (arrow).
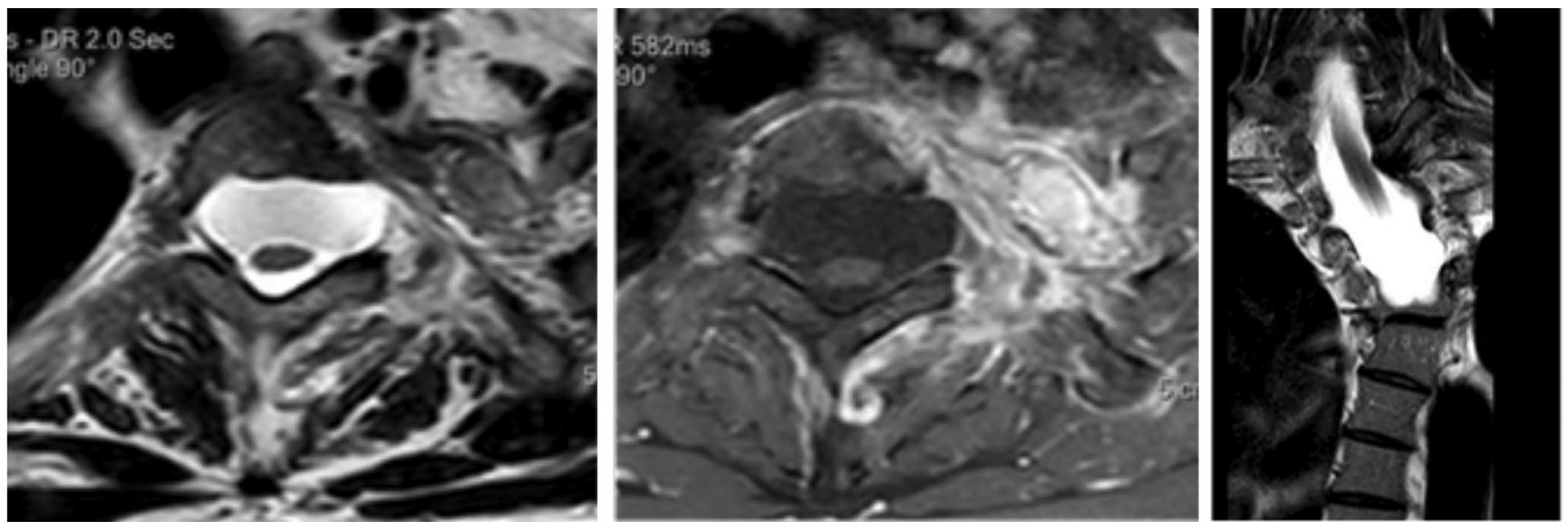

Figure 3. Thoracic MRI: dural ectasia absence of neurofibromas. 
tesis which revealed an uncoagulable hemorrhagic fluid. Thoracic CT angiography, showed a right pleural effusion of great abundance, seat of spontaneously hyperdense zones without image of extravasation of contrast product (Figure 5). Thoracic drainage allowed evacuating $1000 \mathrm{ml}$ of hemorrhagic fluid. Because of a deglobulization at $5.4 \mathrm{~g} / \mathrm{dl}$ noted after $12 \mathrm{~h}$ of surveillance (initial hemoglobin at $12.5 \mathrm{~g} / \mathrm{dl}$ ), video-assisted thoracoscopic hemostasis surgery was performed. Pleural exploration showed a pleural hematoma next to the $5^{\text {th }}$ intercostal space, as well as an active bleeding at this level. Electrocoagulation and clot removal were performed. Patient's evolution was favorable.

\section{Discussion}

Spontaneous hemothorax is a rare and potentially fatal complication of NF1 that may reveal vascular manifestations of the disease in vessels of different sizes

When large vessels are affected, subclavian and intercostal arteries are most often involved and are often the site of stenosis or aneurysm [4]. In other cases, small vessels are responsible for intra-thoracic bleeding.

Arterial involvement in patients with NF1 is well described in the literature but is found very rarely in clinical practice. Its incidence is 1 to $3 \%$ [3]. This value is underestimated because of the silent nature of most lesions and the inaccessibility of certain vessels to clinical examination [5]. All arteries could be affected. This may include stenosis, aneurysm, and angiodysplasia [3]. Several theories have been advanced to better understand the pathogenesis of vasculopathies in NF1. A first hypothesis stipulated that vascular involvement could be attributed to intimal thickening secondary to Schwann cells' proliferation by analogy to the pathogenesis of neurofibromas characterizing this disease [6]. More recent studies suggested that vasculopathy is rather due to an abnormal proliferation of smooth muscle cells present in the vascular media. This proliferation is responsible for the alternation of stenosis and aneurysms, making the vessel wall fragile and vulnerable $[7,8]$.

Another theory was that during NF1, vasculopathy could be secondary to the alteration of normal process of vascular repair. It might be due to neurofibromin's dysfunction which have a tumor suppressing role [2]. Indeed, the dysregulation of this protein could induce excessive migration and proliferation of smooth muscle cells in response to changes in the vascular wall, particularly atherosclerotic plaques $[7,8]$. This would explain the superposition of atheromatous plaque mapping and dysplastic vascular lesions of NF1 reported by some authors $[3,7,8]$.

Hemorrhagic manifestations could also be favored by abnormalities of primary hemostasis (intrinsic platelet abnormality, collagen abnormality, reduction of factor XII, decrease in Willebrand factor) encountered during NF1 [4].

For both patients, a thoracic CT-scan was performed to localize the origin of the bleeding. Careful analysis of intrathoracic arteries including subclavian, intercostal and internal mammary arteries did not reveal any evidence of contrast extravasation or anomaly of vessels' s contour or caliber. Surgical exploration of our 2 patients did not find any anatomical substratum at the origin of bleeding. Our

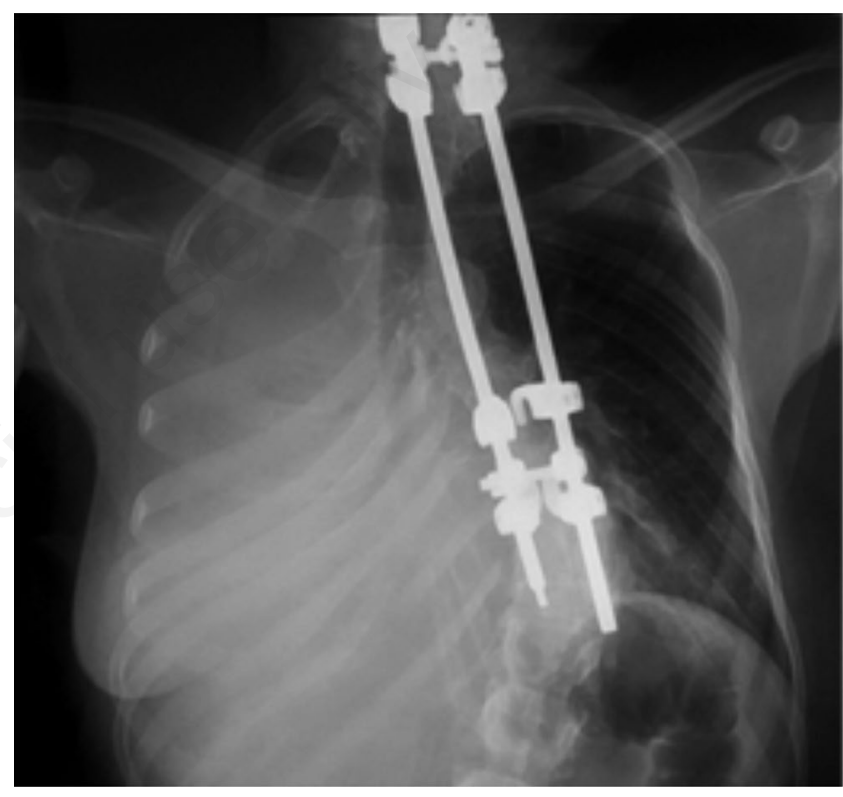

Figure 4. Chest X-ray: right pleural effusion of great abundance.
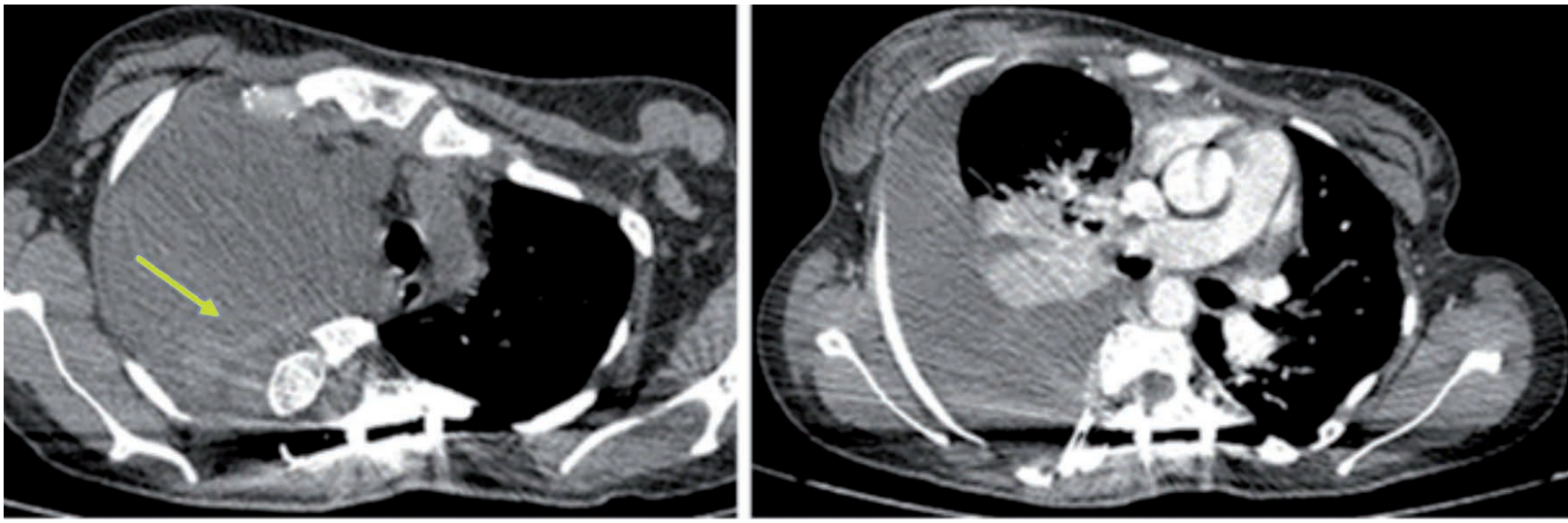

Figure 5. Chest CT scan before and after contrast injection: right pleural effusion with hyperdense component (hemothorax) (arrow). No evidence of arterial injury or mesenchymal tumor. 
explanation was the rupture into the pleural cavity of small dysplastic vessels without the possibility of identifying them by angio-CT. Our two patients underwent video-assisted thoracoscopy, immediately for the first and after chest drainage for the second. Our two patients evolved favorably without recurrence of intrathoracic hemorrhage. Management of spontaneous hemothorax during NF1 is not consensual and depends on several factors: hemodynamic instability or bleeding recurrence requiring in most cases an emergency surgical exploration. Identifying a vascular anomaly in CT angiography could lead to an endovascular embolization [9]. Video-assisted thoracic surgery was considered the gold standard [10]. Some authors propose however a conservative treatment consisting only of a thoracic drainage, especially for patients who are clinically stable without any radiological (angio-CT or angiography) anomaly [4,7].

\section{Conclusions}

Spontaneous hemothorax during NF1 is an uncommon but potentially fatal complication. The origin of bleeding can be identified in some cases, allowing radical treatment by embolization or surgical ligature. In other situations, imaging does not reveal any vascular abnormality. It is likely to be a small vessel 's disease involvement. Therapeutic approach is not consensual.

\section{References}

1. Zamora AC, Collard HR, Wolters PJ, et al. Neurofibromatosisassociated lung disease: a case series and literature review. Eur Resp J2007;29:210-4.

2. Pinson S, Wolkenstein P. [Neurofibromatosis type 1 or Von Recklinghausen's disease]. Rev Med Int 2005;26:196-215.

3. Elmesnaoui A, Benlahbib M, Lekehal B, et al. [L'atteinte des artères périphériques au cours de la neurofibromatose de Von Recklinghausen. Étude de deux cas].[Article in French]. J Malad Vascul 2011;36:189-95.

4. Pulivarthi S, Simmons B, Shearen J, Gurram MK. Spontaneous hemothorax associated with neurofibromatosis type I: A review of the literature. J Neurosci Rural Pract 2014;5:269-71.

5. Pezzetta E, Paroz A, Ris HB, Martinet O. Spontaneous hemothorax associated with von Recklinghausen's disease. Eur J Cardiothorac Surg 2003;23:1062-4.

6. Salyer WR, Salyer DC. The vascular lesions of neurofibromatosis. Angiology 1974;25:510-9.

7. Fdil S, Bouchikhi S, Bourkadi J-E. [Hémothorax spontané: complication rare de la neurofibromatose type 1].[Article in French]. Pan Afr Med J 2017;28:85.

8. Hamilton SJ, Friedman JM. Insights into the pathogenesis of neurofibromatosis 1 vasculopathy. Clin Genet 2000;58:341-4.

9. Ben Saad S, Abdenadher M, Attia M. [Un hémithorax blanc chez une jeune portant des tâches café au lait].[Article in French]. Rev Pneumol Clin 2018;74;100-3.

10. Vaziri M, Mehrazma M. Massive spontaneous hemothorax associated with Von Recklinghausen's disease. Ann Thorac Surg 2006;82:1500-1. 